\title{
The Crystal Eye $X$ and gamma ray detector for space missions
}

\author{
F. Barbato, ${ }^{a, b, *}$ A. Abba, ${ }^{c}$ A. Anastasio, ${ }^{d}$ G. Barbarino, ${ }^{d}$ A. Boiano, ${ }^{d}$ R. de \\ Asmundis, ${ }^{d}$ I. De Mitri, ${ }^{a, b}$ L. Ferrentino, ${ }^{c, e}$ F. Garufi, ${ }^{d, e}$ F. Guarino, ${ }^{d, e}$ R. Guida, ${ }^{f} \mathbf{S}$. \\ Papa, ${ }^{f}$ F. Renno ${ }^{f}$ and A. Vanzanella ${ }^{d}$ \\ ${ }^{a}$ Gran Sasso Science Institute, Via Michele Iacobucci 2, L'Aquila, Italia \\ ${ }^{b}$ Laboratori Nazionali del Gran Sasso, Via Giovanni Acitelli 22, Assergi, Italia \\ ${ }^{c}$ Nuclear Instruments SRL, Via Lecco 16, Lambrugo, Italia \\ ${ }^{d}$ Istituto Nazionale di Fisica Nucleare - Sezione di Napoli, Via Cintia, Napoli, Italia \\ ${ }^{e}$ Dipartimento di Fisica "E. Pancini", Università degli Studi di Napoli Federico II, Via Cintia, Napoli, Italia \\ ${ }^{f}$ Dipartimento di Ingegneria Industriale, Università degli Studi di Napoli Federico II, Piazzale Tecchio, \\ Napoli, Italia \\ E-mail: felicia.barbato@gssi.it
}

Crystal Eye is a new concept of all sky monitor for the observation of $10 \mathrm{keV}-30 \mathrm{MeV}$ cosmic photons exploiting a new detection technique, which foresees enhanced localization capability with respect to current instruments. This is now possible thanks to the use of new materials and sensors.

The proposed detection module is designed to be easily installed either on free flyer satellites or onboard space stations. Science goals include Gamma Ray Bursts, electromagnetic counterpart of Gravitational Wave emissions, Active Galactic Nuclei and line emission from supernova explosions observations. A Crystal Eye pathfinder, made by 4 pixels, has been set up to fly aboard of the Space RIDER, an uncrewed reusable orbital spaceplane aiming to provide the European Space Agency (ESA) with affordable and routine access to space.

The mission will follow a LEO orbit ( $400 \mathrm{~km}, 5.3^{\circ}$ of inclination) for two months then it will come back at the base. We here present the Crystal Eye detection method and characteristics and the first characterization of the pathfinder.

$37^{\text {th }}$ International Cosmic Ray Conference (ICRC 2021)

July 12th - 23rd, 2021

Online - Berlin, Germany

\footnotetext{
*Presenter
} 


\section{Introduction}

Cosmic $\mathrm{X}$ and gamma photons are powerful probes for phenomena occurring in extreme conditions in the Universe and their observations with space-based high resolution instruments can impressively contribute to our understanding of Nature. Up to now, we had wonderful experiments (e.g. Fermi, Chandra, BeppoSax) providing impressive results and important discoveries in the hard $\mathrm{X}$-ray and low energy gamma range $(\mathrm{E} \sim 10-200 \mathrm{keV})$ and high energy gamma rays range ( $\mathrm{E}$ $1 \mathrm{GeV})$. Nevertheless, the medium energies $(\mathrm{E} \sim 1 \mathrm{MeV})$ in the gamma sky are still under-explored. Recently, two important observations (i.e. GW170817 [1] and TXS0506+056[2]) cleared the way for the multimessenger astronomy era. FIG.1, shows GRB170817A lightcurves obtained by FERMI and INTEGRAL in correspondence of the observation of the GW170817 event.

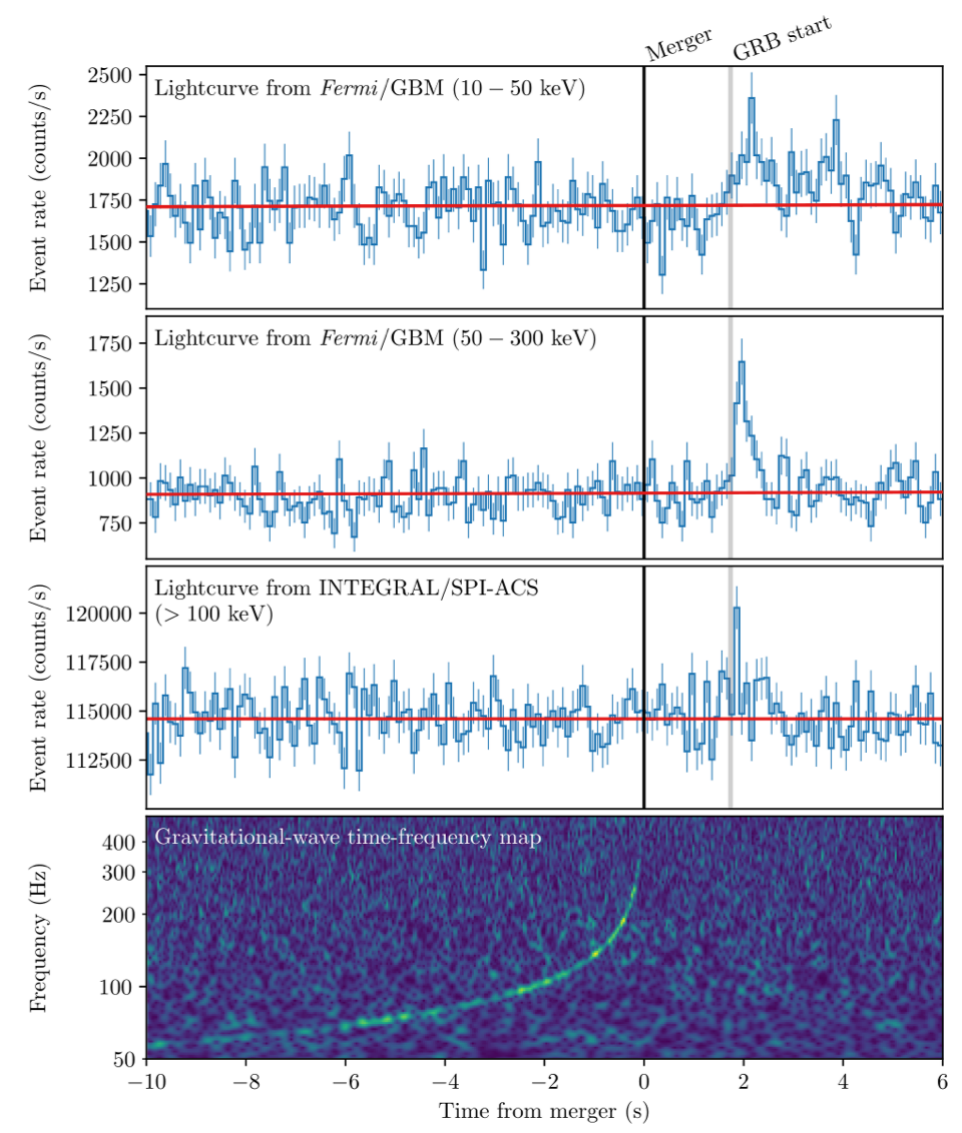

Figure 1: Multimessenger detection of GW170817 and GRB170817A. Top: the summed GBM lightcurve for sodium iodide (NaI) detectors 1, 2, and 5 for GRB 170817A between 10 and $50 \mathrm{keV}$, matching the 100 ms time bins of the SPI-ACS data. Second: the same as the top panel but in the 50-300 keV energy range. Third: the SPI-ACS lightcurve with the energy range starting approximately at $100 \mathrm{keV}$ and with a high energy limit of least $80 \mathrm{MeV}$. Bottom: the time-frequency map of GW170817 was obtained by coherently combining LIGO-Hanford and LIGO-Livingston data. Plot from [3]

FIG.2, instead, shows the broadband spectral energy disrtibution for the blazar TXS 0506+056. The spectral energy distribution is based on observations obtained within 14 days of the detection 
of the IceCube-170922A event. In this very interesting event, observation in the $\mathrm{MeV}$ energy range are missing.

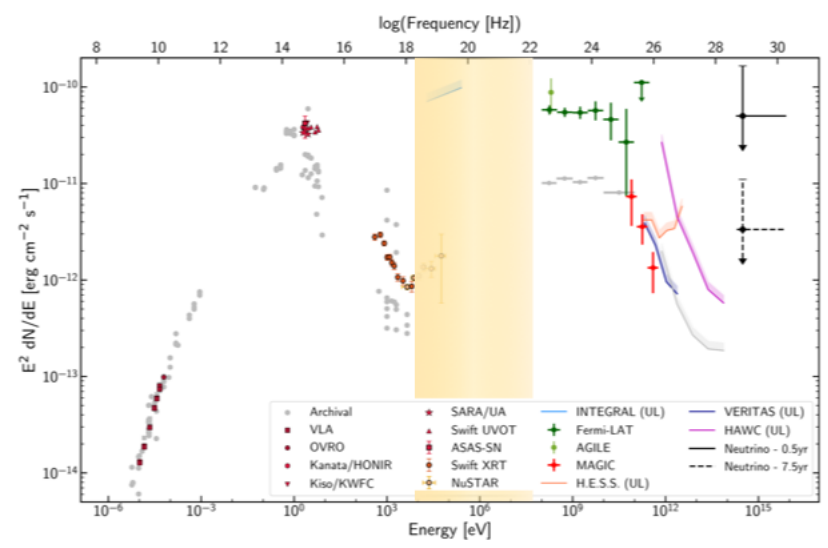

Figure 2: The broadband spectral energy distibution for the blazar TXS 0506+056. Plot from [4]. In yellow the Crystal Eye energy range.

The above mentioned examples are only the most recent that highlight the necessity of new instruments in the gamma medium energy range $(\mathrm{E} \sim \mathrm{MeV})$.

In this panorama is born the Crystal Eye idea. Crystal Eye, indeed, is a proposal for a modular pixelated all sky monitor, that in his baseline configuration is able to observe the gamma sky from $10 \mathrm{keV}$ to $30 \mathrm{MeV}$. The detector, see FIG. 3, has an hemispherical design with two shalls of LYSO crystals and an anticoincidence layer, each read by a SiPM-array [5],[6].

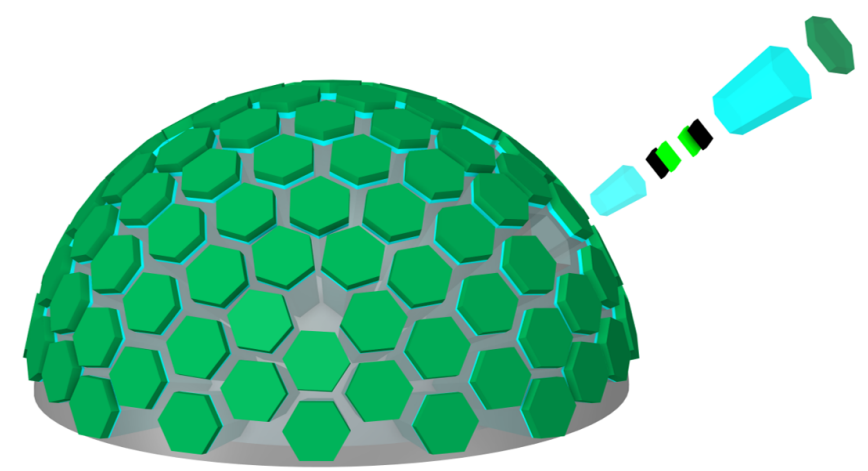

Figure 3: Exploded model of the Crystal Eye detector in the baseline configuration.

It has been designed to work both as free flyer or mounted on space stations.

The Crystal Eye pixel sizes and positions has been chosen in such a way to allow to distinguish among different directions and energy by following the logic in FIG.4 [7]. In this logic, cases A, B and $\mathrm{C}$ are considered to be good events.

\section{The Crystal Eye prototype for Space Rider}

A Crystal Eye pathfinder has been designed and realized to be tested in view of the mission on the Space Rider by ESA. Space Rider is an uncrewed robotic laboratory about the size of two 


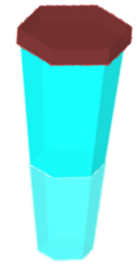

(A)

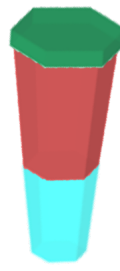

(B)

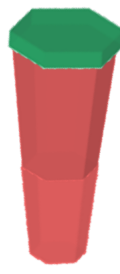

(C)

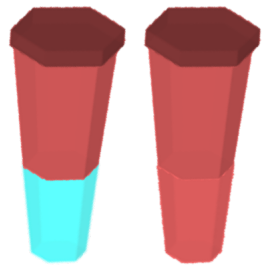

(D)

(E)

Figure 4: Scheme of the Crystal Eye operation principle. In red, the parts with signal. (A) hard X-ray stopping in the anticoincidence. (B) Gamma ray with $\mathrm{E}<1 \mathrm{MeV}$. (C) Gamma ray with E $>1 \mathrm{MeV}$. (D) Low energy charged particle. (E) High energy charged particle.

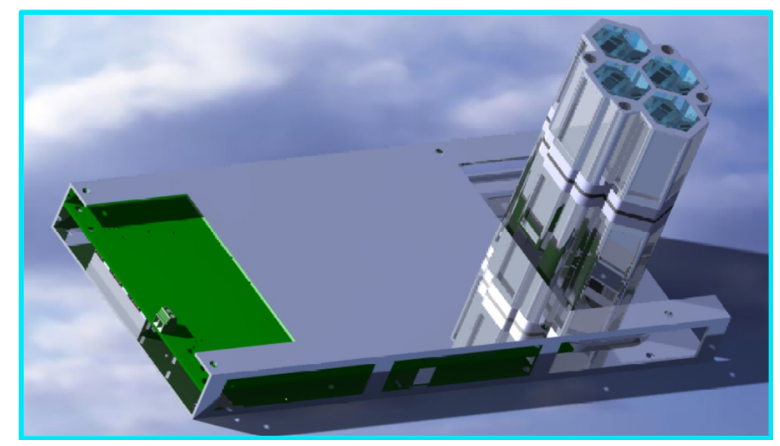

Figure 5: Rendering of the Crystal Eye pathfinder for Space Rider Mission.

minivans. After launch on Vega-C it will stay in low orbit for about two months. At the end of its mission, it will return to Earth with its payloads and will be prepared for a new flight [8].

The Crystal Eye prototype is composed by four pixels, see FIG. 5.

For this prototype LYSO crystals from different brands and with different treatement of the surfaces were used. In particular we use a LYSO pixel made by OST Photonics with grounded surfaces, two LYSO pixels made by Epic Crystals one with grounded surfaces and the other with polished surfaces and Enahnced Specular Reflector (ESR), see FIG. 6.

A fourth temporary pixel made by BGO and manufactured by OST Photonics is also used. Each crystal is read by an MPPC-array S13361-3050-04AE by Hamamatsu Photonics KK. Each array is composed by 16 MPPCs, we read each quadrant by setting an OR circuit among the four anodes and cathodes. This will ensure redoundancy as well as high and low gain channels [9]. All the channels are readout by a custom DAQ board based on the CITIROC1A chip for the signal processing and on the Zynq 7020 for the trigger logic, programmed by Nuclear Instruments SRL [10].

All the pixels are assembled in the temporary 3D printed mechanical support, see 7, in order to test the correct operation of the overall system.

The first measurement gave us information about the LYSO activity and response and solved some concerns about the measurements in [9], and so a very important hint on the best treatment for the surfaces. The MPPC-arrays are all working at $2 \mathrm{~V}$ overvoltage.

The plot in FIG. 8 tell us that OST Photonics crystals produce more light than Epic Crystals, 


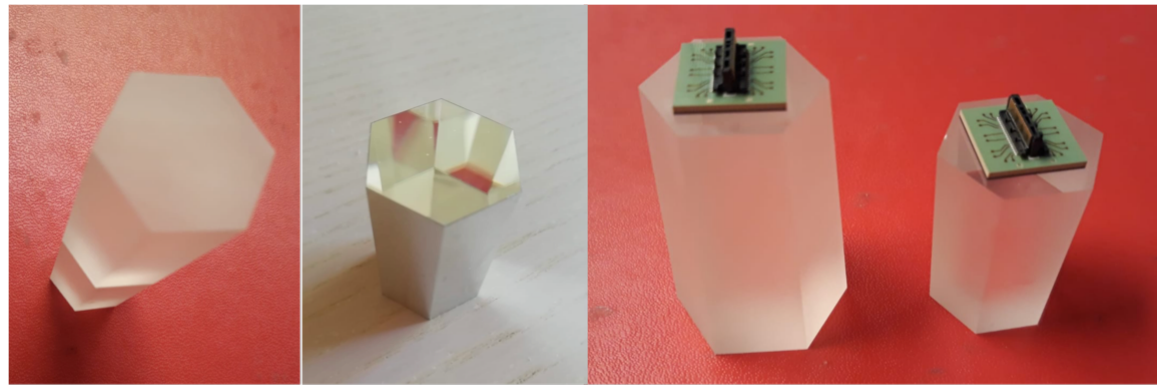

(A)

(B)

(C)

Figure 6: Pictures of the LYSO crystals. (A) OST Photonics Up and Down pixels. (B) Down Pixel with ESR by Epic Crystals. (C) Up and Down pixel with grounded surfaces by Epic Crystals each coupled with its MPPC array.

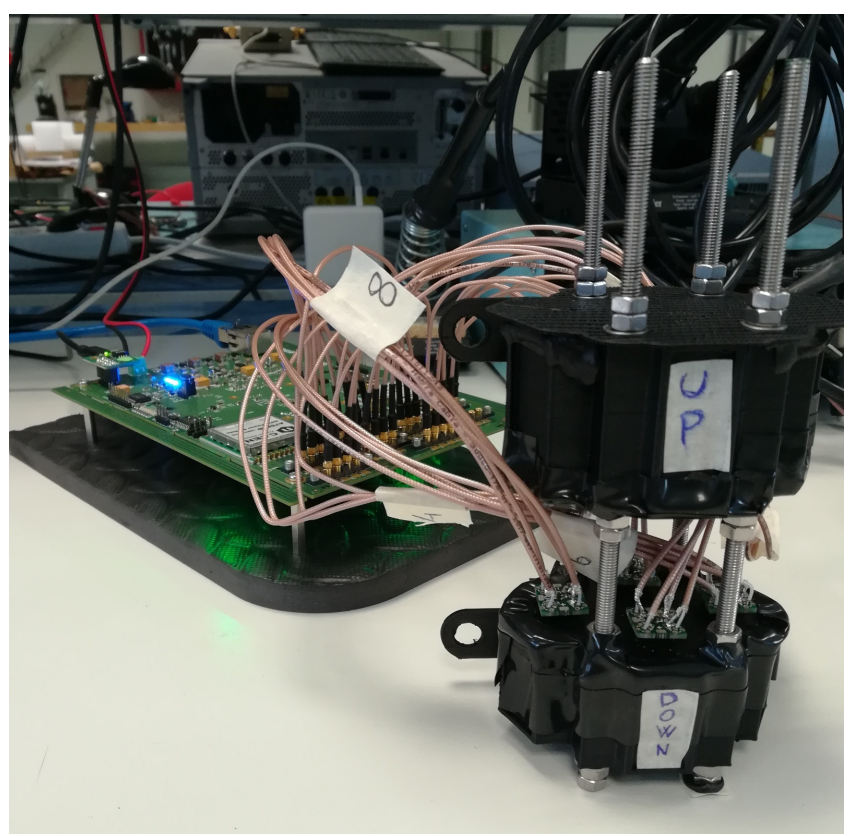

Figure 7: Picture of the assembled prototype.

considering the same size and surface treatment. Moreover, it is also evident that the LYSO crystal by Epic Crystals with polished surfaces and ESR shows a higher light response with respect to the others. We can, therefore, assume that a LYSO with polished surfaces and ESR made by OST Photonics would be the better choice in terms of photons collected by the MPPC-array.

Further measurements with radiactive sources have been made to test the AND logic of the DAQ. Nevertheless, the non-optimized mechanical support was obstructing this test. A new optimized mechanical support is going to be 3D printed at the Additive Manufacturing Office in Laboratori Nazionali del Gran Sasso (LNGS). 


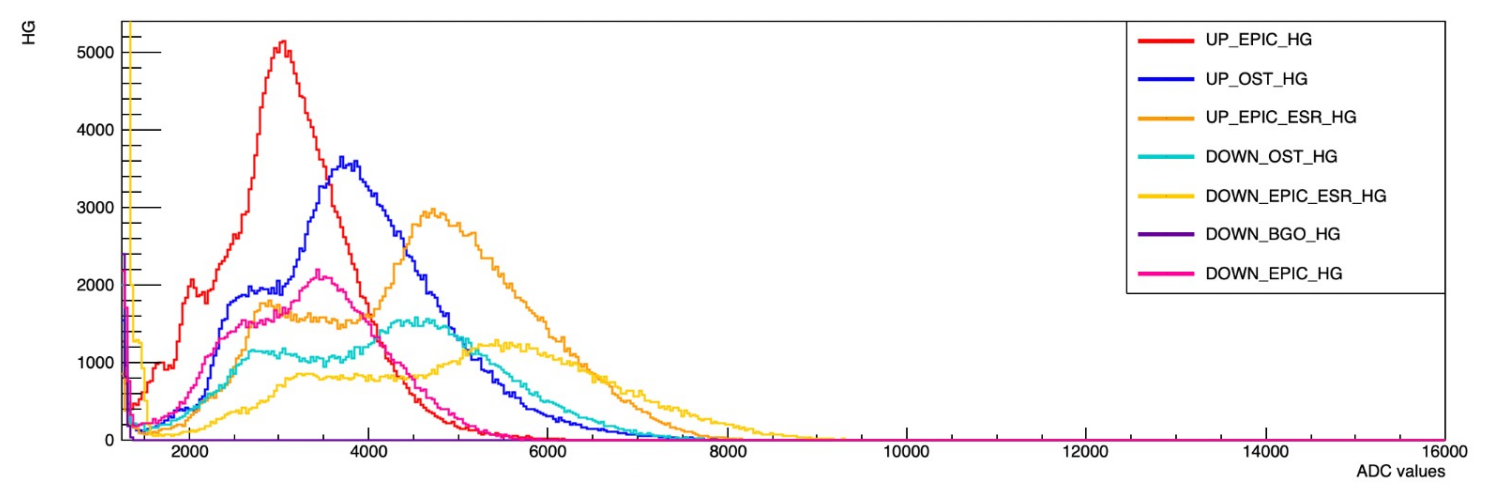

Figure 8: Plot of the LYSO activity of the 3 pixels. On the $\mathrm{X}$ axis there are the ADC values. On the $\mathrm{Y}$ axis the counts obtained in the high gain (HG) channel.

\section{Conclusions and perspectives}

The Crystal Eye is a new concept for an hard X-ray and gamma ray detector designed to fly on LEO orbits as free flyer or mounted on space stations. The main scientific object will be to contribute to the study of gamma-ray physics in the $\mathrm{keV}-\mathrm{MeV}$ energy range. We expect from such a design to obtain a better localization capability with respect to currently orbiting all sky monitors. A pathfinder is expected to fly on the Space Rider by ESA in 2023 for two months. The mission is aimed at testing in the space environment the LYSO crystals, the MPPC-arrays and the DAQ board. Moreover we will have the chance to characterize the background at the Space Rider orbit.

A prototype of the pathfinder has been built to check the correct operation of the overall system. A very important hint on the best LYSO crystal and surface treatment was given by these measurements. Further measurements with an optimized mechanics are ongoing. After these tests, the prototype will start the space qualification phase.

\section{Acknowledgments}

The Crystal Eye project is financed by University of Naples and Intesa San Paolo Bank with the competitive call "Bando STAR2018 - L1 Junior Principal Investigator" and by Gran Sasso Science Institute.

Authors want to thank Paride and Riccardo D'Incecco for prototyping the temporary mechanical support.

\section{References}

[1] Abbott, Benjamin P., et al. "GW170817: observation of gravitational waves from a binary neutron star inspiral." Physical Review Letters 119.16 (2017): 161101.

[2] Aartsen, Mark, et al. "Neutrino emission from the direction of the blazar TXS 0506+ 056 prior to the IceCube-170922A alert." Science 361.6398 (2018): 147-151. 
[3] Abbott, Benjamin P., et al. "Gravitational waves and gamma-rays from a binary neutron star merger: GW170817 and GRB 170817A." The Astrophysical Journal Letters 848.2 (2017): L13.doi:https://doi .org/10.3847/2041-8213/aa920c

[4] The IceCube, Fermi-LAT, MAGIC, AGILE, ASAS-SN, HAWC, H.E.S.S., INTEGRAL, Kanata,Kiso, Kapteyn, Liverpool telescope, Subaru, Swift/ NuSTAR, VERITAS and VLA / 17B-403 teams, Multimessenger observations of a flaring blazar coincident with highenergy neutrino IceCube-170922A, Science 361, eaat1378 (2018), doi:https://doi .org/ $10.1126 /$ science . aat 1378

[5] Renno, F., et al. "Ahp and multi-voting approaches for conceptual design of new detectors: The crystal eye case study." (2020): 470-476.

[6] Renno, F., et al. "Systems Engineering approach for the concept design of the Crystal Eye detector." International Journal on Interactive Design and Manufacturing (IJIDeM) 15.1 (2021): $81-84$.

[7] F. C. T. Barbato, G. Barbarino, A. Boiano, R. de Asmundis, F. Garufi, F. Guarino, R. Guida, F. Renno, and A. Vanzanella "Crystal Eye: a wide sight on the Universe looking for the electromagnetic counterpart of gravitational waves", Proc. SPIE 11118, UV, X-Ray, and Gamma-Ray Space Instrumentation for Astronomy XXI, 111181J (9 September 2019); https://doi.org/10.1117/12.2529095

[8] https://www.esa.int/Enabling_Support/Space_Transportation/Space_Rider

[9] F. C. T. Barbato, G. Barbarino, A. Boiano, A. Vanzanella, F. Garufi, F. Guarino, F. Renno, S. Papa, R. Guida, F. Di Capua, "Preliminary results of the pixel characterization for the Crystal Eye, a new x- and y-ray satellite detector for multi-messenger astronomy," Proc. SPIE 11444, Space Telescopes and Instrumentation 2020: Ultraviolet to Gamma Ray, 114449A (13 December 2020);https://doi . org/10.1117/12.2575885

[10] L. Ferrentino, "R\&D dell'elettronica di DAQ per il volo del pathfinder del rivelatore Crystal Eye", Master Thesis in Physics, Università degli Studi di Napoli Federico II (2021) http://www. fisica.unina.it/documents/12375590/13725484/ 3145_FerrentinoL.pdf/f815b4a1-6c5d-428a-b001-e00280e47672 